\title{
Projekt-Workshop „Sprechtempo und Reduktion im Deutschen“ an der Universität Leipzig vom 13.-14. Juni 2019
}

$\mathrm{Ob}$ ein Mensch langsam oder schnell spricht, ob deutlich oder undeutlich, meinen wir subjektiv zumeist einigermaßen leicht beurteilen zu können. Sprechgeschwindigkeit und phonetische Reduktion empirisch reliabel zu bestimmen, erweist sich jedoch bei genauerer Betrachtung als eine hochkomplexe Aufgabenstellung. Dies findet seinen Niederschlag nicht zuletzt in der Tatsache, dass hierzu zwar bereits umfangreiche problembeschreibende Literatur existiert, aber wohl keine der bislang publizierten Messmethoden für sich in Anspruch nimmt, eine grundlegende Lösung darzustellen.

An der Universität Leipzig beschäftigt sich seit 2018 das Projekt „SpuRD - Sprechtempo und Reduktion im Deutschen" mit diesem Phänomenkomplex, also mit der Frage wie Sprechgeschwindigkeit und phonetische Reduktion - insbesondere vor dem Hintergrund regionaler Distributionen - miteinander zusammenhängen. Für den 13. und 14. Juni 2019 waren ausgewiesene Experten zu einem Projekt-Workshop eingeladen, der sich neben der zentralen Frage der Messbarkeit von Sprechgeschwindigkeit und phonetischer Reduktion auch mit Aspekten der kartografischen Darstellbarkeit dieser Phänomene widmete. Neben den eingeladenen Referenten bestand das Auditorium aus einer kleinen Zahl rein ,passiver" Teilnehmer, so dass intensive und problemlösungsorientierte Diskussionen möglich waren. Es zeigte sich im Verlauf des Workshops einmal mehr, dass die metrische Erfassung gesprochener Sprache mit vielerlei Schwierigkeiten behaftet ist, für die bislang kaum befriedigende Lösungen existieren. Beschlossen wurde der Workshop mit einer substanziellen und ausgedehnten Schlussdiskussion, in der es gelang, verschiedene konkrete neue Strategien für das Projekt herauszuarbeiten und zu initiieren.

Der erste Tag begann mit einer Begrüßung durch Beat Siebenhaar (Universität Leipzig), der sich hocherfreut zeigte über das Zustandekommen des Workshops. Im Anschluss gab Matthias Hahn (Universität Leipzig) eine knappe Einführung in das Projekt, zu dem der Workshop Hilfestellungen leisten sollte, ebenso wie zu den vorläufigen Ergebnissen und den sich abzeichnenden methodischen und technischen Problemen. Hahn führte aus, dass es zunächst um die generelle Frage gehe „Wer spricht wo wie schnell?“. Für verschiedene deutsche Dialekte existierten bekanntlich Stereotype hinsichtlich ihrer generellen Sprechgeschwindigkeit. Diese sollten kritisch hinterfragt und auf Grundlage einer reliablen Datenbasis überprüft werden. Das Projekt bewege sich damit im Spannungsfeld der drei Termini „Sprechgeschwindigkeit“, „phonetische Reduktion“ und „Sprachraum“ und versuche diese miteinander zu kombinieren. Hierbei sehe man sich in der bereits existierenden Literatur mit ,unfassbar vielen terminologischen und methodischen Problemen" konfrontiert und hoffe, mithilfe des Workshops eine belastbare Methodik weiter fundieren und ausbauen zu können. Zugleich sei Literatur zur Beziehung von phonetischer Reduktion zum Sprachraum ein völliges Desiderat, das dringend der Bearbeitung bedürfe. 
Im Folgenden präsentierte Hahn einen Satz von Arbeitshypothesen, die der Arbeit des Projektes zugrunde liegen: Globale Sprechgeschwindigkeit variiert in der Standardaussprache abhängig von der dialektalen Region. Dies resultiert aus den existierenden regionalsprachlichen Varianten. Qualität und Quantität konkreter phonetischer Reduktionen sind abhängig von Sprachgeschwindigkeit. Erhöhte Sprechgeschwindigkeit verstärkt regionale Charakteristika. Es gibt unterschiedliche Strategien, wie Sprechgeschwindigkeit erhöht werden kann: Komprimierung des phonetischen Materials bei wesentlicher Beibehaltung aller Segmente oder Preisgabe phonetischen Materials - Reduktion - bei weitgehender Beibehaltung der zeitlichen Ausdehnung der verbleibenden Elemente. Als Datengrundlage für die Untersuchungen des Projekts findet Äsop-Fabel „Nordwind und Sonne“ aus dem Korpus „Deutsch heute“ des Leibniz-Institut für Deutsche Sprache in Mannheim Verwendung. Diese ist für jeden Sprecher sowohl in normaler wie in hoher Vorlesegeschwindigkeit im Korpus enthalten. Aus dem kontrastiven Vergleich dieser beiden Varianten soll eruiert werden, welche Strategien zur Erhöhung von Sprechtempo Verwendung finden, sowohl global als auch ggf. für einzelne dialektale Regionen oder gar einzelne Sprecher. Betrachtet werden verschiedene, jeweils an die Sprachregion angepasste Phänomene, die hinsichtlich Reduktionsstrategien als besonders aussagekräftig gelten können, so beispielsweise r-Vokalisierung, Kontraktion von Konsonantenclustern, Degeminierung (bei Sprechern aus der Schweiz). Dieses Vorgehen trage der Hypothese Rechnung, dass je nach Sprachregion unterschiedliche Reduktionsstrategien anzunehmen sind. Hahn skizzierte im Anschluss die bislang geplante Analysestrategie des Projektes. Diese bestehe vereinfacht aus dem Dreischritt Segmentierung, Einlesen in eine Datenbank und Generierung von Karten. Hierbei gebe es für alle drei Schritte substanzielle methodische Fragen, zu denen man sich im Rahmen dieses Workshops neue Impulse erhoffe. Als ersten Eindruck stellte Hahn einige vorläufige Ergebnisse und Karten vor, die auf einer reduzierten Datenbasis beruhen. So konnte er berichten, dass es für einzelne Sprecher zwischen normaler und schneller Lesegeschwindigkeit für den Gesamttext eine Zeitdifferenz von etwa 20 Sekunden gebe. Hinsichtlich eines verbreiteten phonetischen Phänomens wie der Elision ganzer phonetischer Segmente zeige sich bereits jetzt, dass Sprecher des west- und nordwestdeutschen Raumes in einem höheren Maße hiervon Gebrauch machen als Sprecher aus dem Osten und Südosten Deutschlands. Dies sei besonders bemerkenswert, da gerade erstere Regionen ja als dem Standarddeutschen näher angesehen werden. Als bisheriges Zwischenfazit des Projektes lasse sich festhalten, dass Sprechtempo und Reduktionsstrategien tatsächlich regional variierten, die - teilweise klar dialektal basierten Raumstrukturen hingegen seien über beide Konditionen weitgehend stabil. Zum Ende seines Einführungsvortrages präsentierte Hahn eine Zusammenstellung verschiedener konkreter Fragen, zu denen man sich im Rahmen des Workshops neue Ansätze und Anregungen erwarte. Hierbei gehe es vor allem um ein objektives Maß für Sprechgeschwindigkeit, aber beispielsweise auch um die Frage, wie sich die Befunde zugleich repräsentativ und kontrastiv in einer Kartendarstellung so visualisieren lassen, dass es nicht zu Verzerrungen kommt.

Im Anschluss stellte Adrian Simpson (Universität Jena) eine Untersuchung zu „Geschlechtsspezifischen Aspekten phonetischer Reduktion“ im Deutschen vor. Ähnliche Untersuchungen zu anderen Sprachen konnten bereits feststellen, dass sowohl die Vokalals auch die gesamte Äußerungsdauer bei Frauen höher ist als bei Männern und erstere 
zudem stärkere Vokalkontraste zeigen. Auch hinsichtlich des Reduktionsverhaltens gebe es bereits Untersuchungen, die für Frauen geringere Werte zeigten. In der vorgestellten Untersuchung wurden Kontraste hinsichtlich Dauer und Reduktionsprozessen auf Lautund Silbenebene untersucht. Datengrundlage waren Aufnahmen von Studenten, für die die Realisierung des intervokalischen Plosivs /g/ in einem lesesprachlichen Kontext untersucht wurde. Zwar zeige sich hinsichtlich der Artikulationsdauern kein statistisch signifikanter Unterschied, jedoch seien die der weiblichen Sprecherinnen tendenziell länger, während die kürzesten Dauern ausschließlich von männlichen Sprechern stammten. Zudem seien $74 \%$ der /g/-Realisierungen von Frauen tatsächlich plosivisch, während es bei den Männern lediglich 40\% seien. Frauen zeigten auch in dieser Untersuchung also insgesamt höhere Artikulationsdauern bei geringerer phonetischer Reduktion. In der anschließenden Diskussion thematisierte Simpson ergänzend zwei grundsätzlichere Fragen, die auch für das SpuRD-Projekt maßgeblich seien: Es gelte zum einen zu klären, bis zu welcher Grenze man die phonetische Realisierung eines phonologischen Plosivlauts noch als Plosiv (im Kontrast zu Frikativ oder Approximant) bezeichnen könne und zum anderen, nach welcher Gesetzmäßigkeit man die Existenz einer Silbe konstatiere. Als Beispiel benannte Simpson die phonetische Realisierung des Wortes <Tagebuch $>$ als ['ta:gbu:x]. Sei an der Nahtstelle zwischen velarem und bilabialem Plosiv noch eine Lösung des ersteren wahrnehmbar, so müsse dies als Korrelat des elidierten Schwa-Lautes und somit als Indiz für eine intendierte Dreisilbigkeit gelten. Sei eine solche Lösung nicht vorhanden, könne von intendierter Zweisilbigkeit ausgegangen werden.

Im dritten Vortrag des Vormittages stellte Jürgen Trouvain (Universität des Saarlandes) seine Untersuchungen zu „Sprechgeschwindigkeit und Pausen in verschiedenen Sprechstilen" vor. Auch er wies nachdrücklich darauf hin, dass es sich bei Sprechtempo und Pausen - insbesondere in der Spontansprache - um ein phonetisch hochkomplexes Thema mit nicht standardisierter Methodik handele, bei dem zudem die bestehende Terminologie bislang sehr heterogen verwendet werde. Daher gelte es, klar zwischen dem eher unpräzisen Oberbegriff „Sprechtempo“ und den enger gefassten Termini „Sprechgeschwindigkeit" und „Artikulationsgeschwindigkeit" $\mathrm{zu}$ differenzieren. Alle in diesem Zusammenhang verwendeten Messgrößen (Wörter pro Minute, Silben pro Sekunde, Laute pro Sekunde) seien problematisch, es müsse für jeden konkreten Fall neu abgewogen werden, welche die geeignetste sei. Der nächste Problemkomplex betreffe den Begriff der Pause, der ebenfalls definitorisch nicht eindeutig gefasst sei. Anerkannt sei allenfalls die grobe Definition als Unterbrechung des Artikulationsflusses, wobei aber über die Mindestdauer einer solchen Unterbrechung bereits keine Einigkeit mehr bestehe. Dies werde spätestens dann problematisch, wenn ein Verschlusslaut an eine Pause grenze. Als weitere Probleme benannte er die in der Spontansprache sehr häufig auftretenden sog. ,gefüllten Pausen“. Auch hier müsse von Fall zu Fall entschieden werden, ob man solche inhaltlich nicht zur Äußerung gehörenden Teile als Pausen definiere. Die im Kontrast hierzu sog. „stillen Pausen“ seien aus phonetischer Sicht häufig nicht tatsächlich still als vielmehr durch Atemgeräusche, Zungenschnalzen und andere potenziell nonverbale Äußerungselemente geprägt. Trouvain wies auch darauf hin, dass zwischen ,tatsächlichen" und perzipierten Pausen ein wesentlicher Unterschied bestehe, da für die Perzeption Faktoren wie Stimmqualität, phonetische Partikeln oder die finale Dehnung von höherer Relevanz seien als Dauerwerte. Sprechtempo und Pausen und ihre Wahrneh- 
mung seien also von sehr vielen Parametern abhängig und ihre Messung dadurch sehr schwer operationalisierbar. In der anschließenden Diskussion wurde unter anderem darauf hingewiesen, dass es nicht ohne weiteres möglich sei, eine anhand von Lesesprache entwickelte Terminologie und Methodik auf Spontansprache zu übertragen; es stelle sich sogar die Frage, ob dies überhaupt zulässig sei. Es zeige sich erneut, wie hochspannend, aber zugleich auch hochproblematisch die Phänomene Tempo und Pause in der Sprache sind.

Volker Dellwo (Universität Zürich) stellte in seinem Vortrag eine Studie zur „Komplexität akustischer Korrelate der Sprechgeschwindigkeit" vor, mit der untersucht werden sollte, inwieweit die Verständlichkeit eines Textes und dessen perzipierte Sprechgeschwindigkeit korrelieren. Hierzu verwendete er technisch verfremdete Aufnahmen gesprochener Sprache, bei denen entweder die Intensitäts- oder die Frequenzinformationen maskiert wurden und von Versuchspersonen mit muttersprachlicher, eingeschränkter oder keinerlei Sprachkompetenz bewertet wurden. Hierbei zeigte sich, dass die verfremdeten Stimuli gleichermaßen als schneller wahrgenommen wurden als ihre entsprechenden „,verständlichen“ Pendants. Die Sprachkompetenz der Versuchspersonen hatte keinen Einfluss auf diese Urteile, also wird die Tempowahrnehmung mutmaßlich nicht durch Verständlichkeit bestimmt. Hörer nehmen offenbar stattdessen die Silben- bzw. Segmentrate wahr, wobei die spektrale Information möglicherweise wesentlich ist für die Tempowahrnehmung. Abgeleitet aus dieser Untersuchung machte Dellwo den Vorschlag, die Anzahl realisierter Silben nicht auditiv, sondern über die Maxima der jeweiligen Amplituden-Hüllkurve zu messen.

Christoph Draxler (Universität München) ging auf technische Aspekte einer korpusbasierten Untersuchung gesprochener Sprache ein. Die bislang als Quasi-Standard verwendeten Analyseprogramme sind seiner Ansicht nach nicht mehr zeitgemäß, da sie auf einem unzureichenden Datenmodell basierten, nicht auf parallele Kollaboration ausgelegt und ihre Annotationseinheiten grundsätzlich zeitgebunden definiert seien. Er plädierte stattdessen für vollkommen neue, datenbankgestützte Analyseprozesse, die strikt zwischen Daten und Anwendung trennten. Es sei wichtig, langfristig und Tool-unabhängig zu planen und die jeweiligen Datenbestände auch entsprechend aufzubereiten. Beispielhaft stellte er eine relationale Datenbank vor, mit der via SQL Abfragen über mehrere - teilweise mit über 130.000 Äußerungen von 1.000 Sprechern sehr große - Korpora möglich sind. Statistische Auswertungen werden mittels externer Statistikpakete durchgeführt, die über standardisierte Schnittstellen auf die Datenbank zugreifen. Als Anwendungsbeispiel präsentierte er Auswertungen von Segmentdauern für Zahlwörter aus den Korpora „Ph@ttSessionz“ und „Deutsch heute“, sowie der regionalen Variation der Dauern von /aI/ in initialer bzw. finaler Wortposition.

Simon Pröll (Universität München) stellte im Anschluss zwei verschiedenartige Methoden zur „Faktorenanalyse und Interpolation in löchrigen Datensätzen“ unter besonderer Berücksichtigung der regionalen Visualisierung von Sprachphänomenen vor. Der konzeptionelle Unterschied zwischen beiden lasse sich in den Formeln „Die Masse hat Recht" und „Nachbarn sind sich einig“ zusammenfassen. In ersterem Fall - der qualitativ-aggregierenden Faktorenanalyse - spiele die Rauminformation keine Rolle, während bei letzterer Methode - der Geostatistik - dies sehr wohl der Fall sei. Pröll demonstrierte das Prin- 
zip der Faktorenanalyse anhand von Beispielen unter anderem aus dem „Atlas der deutschen Alltagssprache" und kontrastierte sie mit dem Verfahren der Clusteranalyse. Hierbei verwies er auf das generelle Problem einer geostatistischen Verfahrensweise, bei der große (d. h. datenreiche) Ortspunkte und Randlagen das Bild verzerrten, und regte eine Diskussion darüber an, inwieweit man solcher Verzerrungen mithilfe verschiedener Kartierungsverfahren Herr werden könne.

Den ersten Vortrag des zweiten Tages bestritt Stefan Kleiner (Leibniz-Institut für Deutsche Sprache, Mannheim) mit einer eingehenden Vorstellung des am Leibniz-Institut für Deutsche Sprache entstandenen Korpus „Deutsch heute“ und exemplarischen Ergebnissen des zugehörigen Projekts „Gesprochenes Deutsch“. Anhand ausgewählter Phänomene zu Aussprache und Betonung einzelner Lexeme demonstrierte er, wie sich mithilfe des Korpus' und der Darstellung von Phänomenen auf Karten regionale Variation innerhalb des deutschen Gebrauchsstandards anschaulich visualisieren lässt. Als ein dominierendes Charakteristikum zeige sich eine häufig auftretende Nord-Süd-Gliederung, deren konkreter Grenzverlauf jedoch je nach Phänomen erheblich variiere. Dieser Befund zeige sich auch bei einer Faktorenanalyse, bei der die Nord-Süd-Verteilung der stärkste Faktor sei. Kleiner verwies darauf, dass die so statistisch gewonnenen Daten große Übereinstimmungen mit ohrenphonetisch (d. h. subjektiv) erhobenen Daten aufwiesen.

Roland Kehrein (Forschungszentrum Deutscher Sprachatlas, Marburg) beschloss den Vortragsteil des Workshops mit einer Darstellung von „Raumbildern in den deutschen Dialekten“. Hierzu stellte er die statistisch basierte Arbeit von Alfred Lameli (2013) der bislang maßgeblichen Darstellung von Peter Wiesinger (1983) gegenüber. Lameli konnte mithilfe seines Similaritätsmodells Wiesingers Befunde grundsätzlich bestätigen, sie zugleich im Detail aber auch deutlich revidieren. Hierzu gehört, dass Lameli nicht wie Wiesinger zwei, sondern fünf dialektale Haupträume identifiziert. Anhand zweier konkreter Phänomene, der Schwa-Apokope und der Realisierung des Präfix' < ge-> zeigte Kehrein abschließend, wie sich auch in standardnaher Sprache noch Elemente einer Restarealität feststellen lassen.

Die intensive Abschlussdiskussion war zunächst von der Opposition von Standardsprache und Dialekt geprägt. Die Teilnehmer waren sich einig, dass die zentrale Frage hierbei sei, was an welchem Ort als Standarddeutsch vermittelt werde und welcher Status diesem Standard und seiner Verwendung jeweils zugeschrieben werde. Hieran schloss sich die Frage an, ob die Begriffe bzw. Konzepte von schneller und langsamer Sprache für das Standarddeutsche und etwaige Dialekte von Sprechern überhaupt vergleichbar angewendet werden und ob entsprechend etwas, das im Standarddeutschen als klares Reduktionsphänomen gelten könne, dies auch im Dialekt sei. Adrian Simpson wies nochmals ausdrücklich darauf hin, dass es zur Silbenwahrnehmung nicht notwendig eines Vokals bedürfe. Es könne stattdessen beispielsweise bereits ausreichend sein, wenn als Reflex des (elidierten) gerundeten Vokals der vorangehende Konsonant hörbar gerundet sei. Seiner Warnung davor, Silbenzahl als eine objektive und zuverlässige Messgröße anzusehen, stimmten andere Teilnehmer ausdrücklich zu, ebenso wie dem Hinweis von Jürgen Trouvain, dass eine hierarchische Gliederung von möglichen phonetischen Reduktionsphänomenen zumindest als hochproblematisch zu bezeichnen sei. 


\section{Literatur}

Lameli, Alfred (2013): Strukturen im Sprachraum: Analysen zur arealtypologischen Komplexität der Dialekte in Deutschland. Berlin/Boston: De Gruyter. (= Linguistik - Impulse \& Tendenzen 54).

Wiesinger, Peter (1983): Die Einteilung der deutschen Dialekte. In: Besch, Werner/Knoop, Ulrich/Putschke, Wolfgang/Wiegand, Herbert Ernst (Hg.): Dialektologie: Ein Handbuch zur deutschen und allgemeinen Dialektforschung (= Handbücher zur Sprach-und Kommunikationswissenschaft 1.2). S. 807-900. Berlin/New York: De Gruyter.

Dr. Fabian Brackhane

Leibniz-Institut für Deutsche Sprache

R5 6-13

68161 Mannheim

E-Mail: brackhane@ids-mannheim.de 